\title{
Topological Inference Through Mobile Devices
}

\author{
Marco Antero Paiva, Marcelo Petry, Rosaldo J. F. Rossetti \\ Artificial Intelligence and Computer Science Laboratory, Department of Informatics Engineering \\ Faculty of Engineering, University of Porto \\ Rua Dr. Roberto Frias, S/N • 4200-465 Porto • Portugal T: (+351) 225081566 F: (+351) 225574103 \\ \{ee04127, marcelo.petry, rossetti\}@fe.up.pt
}

\begin{abstract}
Nowadays location systems are used within a large variety of applications. The application of these systems within indoor environments is already provided by several solutions. However, the need for high accuracy within these environments to pursue such a purpose implies the use of specific infrastructures designed towards it. Our project tries to meet the requirements for a simple, low-cost, and scalable location system through different approaches. The main idea of it is to re-construct topological maps of indoor spaces through location estimation, and to serve as a means of reducing the precision requirements other systems may have to develop a scalable and highly applicable solution.
\end{abstract}

\section{Keywords}

Indoor Localization, Local Positioning Systems, Topology Mapping.

\section{INTRODUCTION}

Location-aware services have become an important area for today's ubiquitous and pervasive computing applications. The increasing use of these kinds of services brought the need to implement them in mobile devices.

Today's solutions provide location-aware services in applications that are oriented towards device's localization in maps that give a metric perception of the environment. Thus, maps are more similar to their real environment. However, we believe that this metric-oriented approach is not the optimum approach towards a scalable implementation of indoor location-aware services used in mobile devices. In this sense, topological mapping approaches bring an abstraction to that metric information and are more likely to deal with low accurate measurements. These two approaches are well studied in the robotics research field, and developed under the SLAM (simultaneous localization and mapping) problematic. While metric maps try to precisely represent the world by measuring and relating distances as it is used for example in [4], the main idea behind the topological approach is to provide distinctiveness for global localization and mapping within the environment as it is done in [2]. A topological map approach can be used also in location-aware services oriented towards human utilization; e.g. humans would not require geometric locations to navigate in indoor environments and could easily do it through semantic locations and connections between

Permission to make digital or hard copies of all or part of this work for personal or classroom use is granted without fee provided that copies are not made or distributed for profit or commercial advantage and that copies bear this notice and the full citation on the first page. To copy otherwise, or republish, to post on servers or to redistribute to lists, requires prior specific permission and/or a fee.

SAC'12, March 25-29, 2012, Riva del Garda, Italy.

Copyright 2012 ACM 978-1-4503-0857-1/12/03 ...\$10.00. them. The same idea can be applied to many intelligent systems which make use of location-aware services.

Our premise is to depart from a system with very little information about the environment. No map and topology is known a priori. Thus, its goal must be to achieve localization in the indoor environment and through that localization start building a topological map of that indoor environment along the time and through a collaborative fashion among users.

\section{BACKGROUND}

The first technology that comes to mind when thinking about localization is the GPS (Global Positioning System). However, this technology has a poor performance in indoor environments due to the lack of satellite coverage. Some works have been carried out also with cellular networks, namely using GSM (Global System of Mobile), but it suffers from the same effects referred to GPS. Nevertheless, better performances can be achieved through the use of a priori data about the environment, such as it was used in [3], through GSM.

One of the most explored technologies to perform localization in indoor environments is the Wi-Fi Wireless LANs. This is a technology widely implemented in public infrastructures and that is also built-in in today's ordinary mobile devices. However, this kind of technology suffers from significant signal attenuation effects in indoor environments. These kinds of problems are usually diminished through fingerprinting approaches, in which an off-line training phase is performed to measure signal characteristics within the environment as it is done for example in [1], one of the most cited solutions. However, this usually leads to lots of a priori setups and data gathering to perform localization. Nevertheless, localization in these environments can obviously be done without fingerprinting, but it implies less accurate estimations. Bluetooth is another sensor technology that can be used towards localization purposes. Despite similar to Wi-Fi in some aspects, Bluetooth measures are only available after establishing a connection between devices, which is not very practical. Another kind of approach can be done using inertial sensors such as accelerometers, magnetometers and gyroscopes. Such an approach is usually made performing dead-reckoning of the devices when the users are on the move through acceleration and orientation measures.

We are specifically interested in studying the potential of available technology to support easy and practical inference of topological maps for indoor application. More specifically, we want to provide some guidelines to select mobile-enabled sensors to base topological map inference methods. Building those topological maps through localization of devices in indoor environments in a collaborative fashion is the long term goal of this project. With the localization estimates of many devices we 
could achieve a better base to implement topological map inference methods.

\section{A PRELIMINARY ASSESSMENT AND RESULTS}

To implement a system which should fulfill the goals of our purpose, we opted to develop a solution using three main sensor technologies towards the localization purpose: Wi-Fi sensors within WLANs performing a lateration technique with a minimum squares optimization procedure, accelerometers and magnetometers sensors to perform dead reckoning with the mobile devices through a pedometer and a compass emulation. This was done using mobile phones running Android OS, which is one of most used operating systems for mobile platforms.

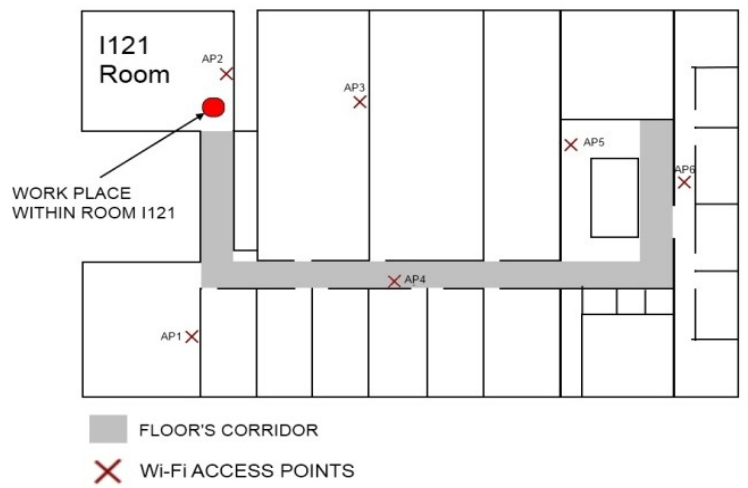

Figure 1. Floor plan where the experiments were made.

The Wi-Fi signal lateration is done through the signal strength of at least 3 Access Points reachable by the devices, and if possible all the reachable ones to improve estimation accuracy. At the same time dead-reckoning is done through measurements of device's acceleration and magnetic field measurements, in order to perform a step count of user movement with pedometer emulation in conjunction with measures of step orientation through the emulation of a compass.

Preliminary results show an accuracy with the Wi-Fi lateration of $6.6 \mathrm{~m}$ with devices stopped, and an error of around $1.41 \mathrm{~m}$, when users are on the move, but with a maximum possible deviation of around $7.37 \mathrm{~m}$, e.g. in relation to the corridor depicted in Figure 1. On the other hand the results obtained with the dead-reckoning procedure show accuracy as low as $1.0 \mathrm{~m}$. The main objection regarding dead-reckoning is that along the time the error increases if no reference point is used to restart the measurement.

With the results we previously presented we are able to conclude that the usage of Wi-Fi lateration estimations when devices are on the move is not sufficiently accurate to provide a good topological map of the environment. To overcome this limitation we use the dead-reckoning estimations when devices are on the move, and we update new reference points when devices are stopped for a long time, with Wi-Fi lateration estimations.

With this approach we are able to build a topological map for example inferring a room, where usually people are stopped for a long time and from there whenever users start walking with the device we perform the inference of the walking path with deadreckoning. In order to identify the static places, we have built a histogram of the Wi-Fi position estimations, and at a certain count higher than a specific threshold, we are able to infer an area to be a static place.
To show an example of that inference procedure we present in Figure 2 the workplace (red circle) depicted in Figure 1 and also the corridor path now inferred through dead-reckoning (blue dots). This was measured when a person working inside room I121 walked through the corridor and came back to its working place. The metric error is noticeable; nonetheless it is possible to observe the topological similarity between the real path (black line in Figure 2), and the inferred one as well as the positioning of the workplace.

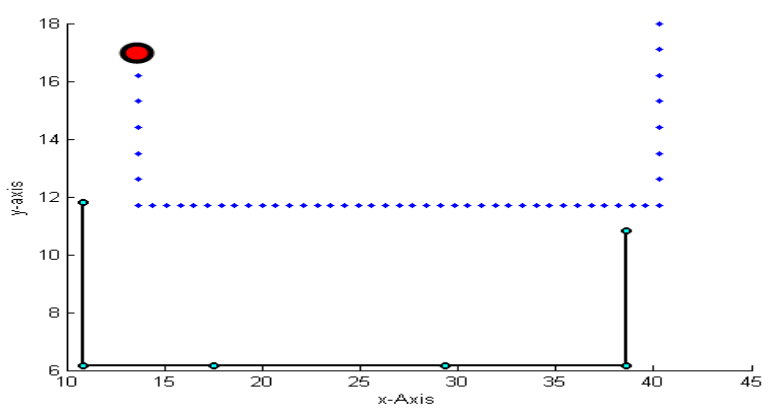

Figure 2. Inference of a room connected to the corridor topology.

\section{CONCLUSIONS}

The main purpose of our work was to develop a system which could provide a tool to perform topology map inference procedures within indoor environments, using mobile computing. We should remark that we were able to prove its feasibility and mainly that a simple, low-cost, and scalable solution can be provided towards that purpose. In this sense analyzing the results we can verify that even with not so accurate and precise results, we can achieve an indoor topology using highly available sensor systems and infrastructures. However, in order to improve this proposed system we should emphasize that with an enhanced inference of the localization estimations we should be able to compute a topology inference through all the sensor technologies at the same time, in opposition to the inference that we made, on which we used the Wi-Fi estimations to infer a room, and performed a dead-reckoning technique to infer the corridor connection to that room.

\section{REFERENCES}

[1] Bahl, P. and Padmanabhan, V. N. 2000. RADAR: an inbuilding RF-based user location and tracking system. In INFOCOM 2000. 19 $9^{\text {th }}$ Annual Joint Conference of the IEEE Computer and Communications Societies, 775-784 vol.772.

[2] Beeson, P., Jong, N. K. and Kuipers, B. 2005. Towards Autonomous Topological Place Detection Using the Extended Voronoi Graph. In Proc. of the IEEE International Conference on Robotics and Automation, ICRA 2005, 4373-4379.

[3] Otsason, V., Varshavsky, A., LaMarca, A. and de Lara, E. 2005. Accurate GSM Indoor Localization. In UbiComp 2005: Ubiquitous Computing, M. Beigl, S. Intille, J. Rekimoto and H. Tokuda Eds. Springer Berlin / Heidelberg, 903-903.

[4] Yiguang, X., Sengupta, R. and Fallah, Y. 2010. Making indoor maps with portable accelerometer and magnetometer. In Ubiquitous Positioning Indoor Navigation and Location Based Service (UPINLBS), 2010, 1-7. 